\title{
Spectroscopic and spectrometric differentiation between abiotic and biogenic material on icy worlds
}

\author{
Kevin P. Hand ${ }^{1}$, Christopher P. McKay ${ }^{2}$ and Carl B. Pilcher ${ }^{3}$ \\ ${ }^{1}$ Jet Propulsion Laboratory, California Institute of Technology, \\ MS 183-601, 4800 Oak Grove Drive, Pasadena, CA 91109 \\ email: khand@jpl.nasa.gov \\ ${ }^{2}$ NASA Ames Research Center \\ Moffett Field, CA 94035 \\ email: chris.mckay@nasa.gov \\ ${ }^{3}$ NASA Astrobiology Institute \\ NASA Ames Research Center, Moffett Field, CA 94035 \\ email: carl.b.pilcher@nasa.gov
}

\begin{abstract}
The ability to differentiate abiotic organic material from material of a biological origin is a critical task for astrobiology. Mass spectrometry and spectroscopy provide key tools for advancing this task and are two techniques that provide useful and highly complementary compositional information independent of a specific biochemical pathway. Here we address some of the utility and limitations of applying these techniques to both orbital and in situ exploration of icy moons of the outer solar system.
\end{abstract}

Keywords. Astrobiology, Europa, Moons, Biosignatures, Exobiology, Spectroscopy.

\section{Introduction}

Astrobiology is the study of the origins, evolution, distribution, and future of life in the Universe, including life on Earth. As with the study of any phenomenon in the Universe - from neutrinos to earthquakes - our knowledge and understanding is limited by our capability to observe and measure the phenomenon in question. The challenge that empiricism imposes has lead to some of the most advanced technical achievements of our civilization, the large hadron collider built by the European Organization for Nuclear Research (a.k.a. CERN) perhaps representing the most extraordinary such achievement to date. In the decades to come, the challenge of astrobiology will continue to push the technological frontier in the pursuit of new knowledge.

As we move forward with our exploration of the Solar System and beyond, the search for, and detection of life on distant worlds persists as one of our top priorities (NRC 2003). For the first time in the history of humanity, we have the technological capability to actively pursue this endeavor. We can now build the spacecraft needed to orbit, land, and investigate the many worlds in our Solar System that may have been, or may currently be, habitable. Nevertheless, our understanding of life as a phenomenon remains largely qualitative and poorly constrained. Indeed, as a phenomenon it lacks a singular universal definition (Cleland and Chyba 2002), though several operational definitions have been proposed (NRC 2003).

Furthermore, the science of biology is not a science based on first principles from which one can then derive an understanding of observations (e.g. as one can do with the Law of Gravity and orbital dynamics). In biology, specific predictions are difficult, though 
Darwinian evolution provides a context for expectations. Given our current understanding of life and the origin of life, the closest we can come to a predictive equation is (Hand et al. 2009),

Liquid water + Biologically essential elements + Energy + Catalytic surface $\rightarrow$ Life

The lack of a definition for 'life' presents considerable challenges for astrobiology. Joyce (1995) humorously notes that the popular definition for life may well be 'that which is squishy'. Of course this definition leaves much to be desired from a measurement standpoint. Though somewhat broad in scope, the 'chemical Darwinian' definition that "life is a self-sustained chemical system capable of undergoing Darwinian evolution" is often offered by biologists as a useful starting point (Joyce 1995; Chyba and McDonald 1995). Darwinian evolution includes three processes: amplification, that is, replication of a genetic description of a prototype; mutation to introduce variation during amplification; and selection of replicates produced during amplification to be used for further amplification (Joyce 1995). This is useful for assessing extant life over time, but when designing an instrument suite for an orbiting or landed spacecraft the prospect of observing Darwinian evolution poses obvious challenges. Furthermore, the detection of life includes extinct life; fossil evidence must be included in our operational definition.

An engineering perspective is useful here. We may think of a circuit containing a battery as an analogue for life. If the circuit is left open a charged battery will slowly lose charge; if a circuit is closed between the terminals, the battery will dissipate energy faster than in the open-circuit scenario. Life closes the chemical and radiation energy circuits available in the environment, advancing the second law of thermodynamics. Depending on the dynamics of the environment, the life structure that provided the 'closed-circuit' can persist in the environment long after the energy has been dissipated, thus providing potential fossils for detection. As presented by McKay (2004) and in detail by Summons et al. (2008) and other workers throughout the history of organic geochemistry, the structures of life arise from a relatively small set of universal building blocks. Thus when we search for life we might be well served by looking for patterns indicative of life's structural biases. McKay (2004) referred to this as the 'Lego principle', in reference to the toy building blocks that come in specified basic structural units.

Ice covered worlds like Europa and Enceladus are highly unlikely to have life on their surfaces, though some interesting concepts have been proposed. Instead, our first dedicated missions to these worlds will need to be able to assess their habitability and characterize in detail their surface compositions. Here we examine the utility of two broad methods, spectrometry and spectroscopy, for differentiation between abiotic and biogenic material on ice covered moons of the outer Solar System. By abiotic we mean compounds and complexity not generated by life. By biogenic, we mean of or derived from life. Europa is presented as the type example of a possibly habitable ice-covered ocean world.

\section{On the Habitability of Europa}

The evidence for a sub-surface ocean on Europa is strong and consistent with a broad range of compositional and geophysical models (Greeley et al. 2004; Schubert et al. 2004). Arguably the most compelling data is the combination of ground-based Doppler RADAR measurements of the Galileo spacecraft, and the magnetometer measurements made from Galileo (Anderson et al. 1998; Khurana et al. 1998; Zimmer et al. 2000). The combination of these observations necessitate a near-surface conducting layer of unit density material below the surface ice of Europa. This set of empirical constraints is hard to satisfy without 
the presence of a salty, global, sub-surface ocean approximately $100 \mathrm{~km}$ thick. Though subject to considerable debate, geological and compositional data also supports the ocean hypothesis (Pappalardo et al. 1998; McCord et al. 1998).

The putative liquid water ocean of Europa is a top priority for future astrobiological investigation (NRC 2003). Though photosynthetic organisms are unlikely due to the thickness of the ice shell (estimated to be a few to $<20 \mathrm{~km}$ in thickness, Hand and Chyba (2007) and references therein), numerous chemosynthetic pathways for life in Europa have been proposed based on models for the availability of the required compounds (Zolotov and Shock 2004). The temperature and pressures predicted for the europan ocean are comparable to those found in the depths of the Earth's oceans, and McCollom (1999) has argued that an active seafloor on Europa could support considerable biomass. If Europa were uninhabited, such hydrothermal activity would nevertheless likely drive the production of organics and other prebiotic compounds on Europa. Key regions of interest for habitability are the ice-water interface of the ice shell and the water-rock interface of the seafloor (Figueredo et al. 2003). Finally, the irradiated surface ice of Europa may also serve as an important source for compounds of both prebiotic and biological interest (Chyba 2000; Kargel et al. 2000; Hand 2007).

Exchange of ocean material with the surface has not been observed on Europa (see e.g. Phillips et al. (2000)). Nevertheless, delivery of oceanic material - and any life or prebiotic compounds contained in the water - to the surface of Europa is hypothesized to occur in several different ways. If the ice of Europa is thin (a few kilometers), fracturing through the entire shell can occur and direct communication between the ocean and surface is possible. In this case, emplacement of material onto the surface would occur through cryovolcanic eruptions and extensional fracturing that exposes fresh oceanic material to the surface. If the ice shell of Europa is thick ( $\sim 15 \mathrm{~km}$ or more) a thin brittle ice shell may overlay a thick convecting ductile ice layer (Pappalardo and Head III 2001). In this case, convective cells within the ductile layer may serve to deliver oceanic material to the base of the brittle ice layer. Tectonic activity in the brittle layer would then be required to bring subsurface material to the surface. Carlson et al. (2009) provide a summary of various mechanisms for such exchange.

The surface of Europa receives an average of $125 \mathrm{~mW} \mathrm{~m}^{-2}$ of energetic electron and ion irradiation (Cooper et al. 2001). This radiation drives chemical modification and weathering of the surface, creating a considerable challenge for differentiating abiotic organic chemistry driven by radiolysis on the surface of Europa from degraded biological chemistry resulting from delivery of oceanic material to the surface. An important aspect of differentiating such exogenous surface chemistry from endogenous biosignatures will be mapping of surface ages and regions of recent activity on Europa. Once such regions are identified based on geological interpretation (e.g. crater counts and monitoring of fractures) then chemical mapping of organic compounds containing nitrogen, phosphorous and sulfur will be a an important step towards identifying possible surface biosignatures of subsurface life.

\section{The detection of Life on Earth}

The technological challenge of detecting life beyond Earth brings into question our capabilities for detecting life on Earth. The development of such technologies, and the extension of such innovations to other worlds, is one of the primary goals of NASA's Astrobiology Program.

The inherent bias, of course, is that many of the tools and techniques to study terrestrial biology are tethered to the specific attributes of life on Earth, which, to the best 
of our knowledge comprises one contiguous genetic tree and one universal biochemistry (that of DNA, RNA, and proteins). Perhaps the best example of this bias is the polymerase chain reaction (PCR) technique that has opened the floodgates to understanding the microbial biosphere. Though revolutionary for the study of RNA/DNA-based life, PCR essentially uses a RNA/DNA baseline to find more RNA/DNA life forms. If you do not know a priori that you might find RNA/DNA-based life, then PCR does you little good. Many other biochemical techniques suffer similar pitfalls.

Mass spectrometry is one of the few in situ techniques that provides useful information on chemical complexity without being tethered to the RNA-DNA-Protein paradigm. The limitation, as discussed in the following section, is that mass spectrometry by itself and without any information on the geological or chemical context of the sample, is not sufficient for answering the binary question 'Is life present?.' If coupled with measurements of isotopic fractionation $\left(e . g . \delta^{13} \mathrm{C}\right)$ and measurements of a chiral excess of one enantiomer (e.g. in amino acids and/or sugars), then a very compelling case can be made for biology having played a role in the production of the sample material.

Detecting life from orbital or airborne platforms presents a host of challenges. Foremost among these challenges is that spectroscopic techniques are largely limited to using photosynthesis as a proxy for the bulk biosphere; very little attention has been given to non-photosynthetic spectroscopic biosignatures. When considering the question of life detection on Europa or Enceladus, one can start with the much more basic question: "Could we detect life on Earth if the ocean was frozen over?" The answer is a resounding 'Yes', but is contingent on photosynthetic cyanobacteria and the absorption features associated with chlorophyll-a, -b, and carotenoids. As discussed below, the use of orbital spectroscopy for the search for life on icy worlds - where photosynthesis is likely a marginal niche, at best - necessitates the identification and development of alternative spectroscopic features to serve as biosignatures for non-photosynthetic life forms. This endeavor advances our capability to search for life beyond Earth while simultaneously enhancing our capability to better monitor life here on Earth.

Finally, spectroscopic and mass spectrometric techniques are highly complementary. Vibrational (i.e. infrared) spectroscopy yields information about the functional groups, branches, and aromatic structure of a given compound. Spectra reveal the relationship of atoms to atoms and groups of atoms to other atoms within a compound. Mass spectrometry reveals the size of the compound and the size of the subunits generated by ionizing the compound (yielding the 'cracking pattern' of the compound). Together, this spectroscopic and mass spectrometric information permit a detailed understanding of the specific compounds under observation.

\section{Spectrometric Signatures}

For over 50 years mass spectrometry has served as a critical tool for assessing the complexity of organic matter (Peters et al. 2005). From oil exploration to the Viking Landers, the ability to measure the mass distribution of sampled compounds provides useful information about the sample composition. When searching for petroleum reserves, the mass distribution provides a metric of thermal maturity and the potential yield of various types of hydrocarbons from a given well. When searching for evidence of life on Mars, the Viking gas chromatograph and mass spectrometer set an upper limit on the concentration of organics in the lander regions - no organic compounds were detected at the parts per billion level (Biemann et al. 1977). Interestingly, however, we note that the Viking experiments were insufficient for detecting the expected background flux from meteorite infall (Benner et al. 2000). 
As a tool for detecting life as we know it, mass spectrometry serves two key roles: 1) detecting organics, and 2) providing information on the complexity of organics. The first yields a binary answer (or rather an upper limit) that informs further investigation. The second yields information that may serve to distinguish abiotic organic chemistry from biologically generated organics. McKay (2004) has argued that the spectrometric distinction can be characterized as the 'Lego Principle'; biology preferentially uses specific organic subunits to build larger compounds while abiotic organic chemistry proceeds randomly. According to the 'Lego Principle', mass spectra associated with life are punctuated by peaks indicative of the subunit preferences while those associated with abiotic chemistry have a broad Gaussian spread of peaks with no obvious structure.

Though the 'Lego Principle' is broadly correct, caution must be taken when comparing abiotic organic chemistry to a rather featureless Gaussian distribution of organics. First, significant differences exist between the processes of abiotic synthesis and that of decomposition of organic material. Breakdown of organic material forms, to varying degrees, Gaussian distributions in mass spectra. As McKay (2004) notes, patterns distinctive of biology are typically lost when exposed to heat and other types of radiation. For example, the degree to which an oil well shows a Gaussian mass spectrum is used in part as a metric of the thermal maturity of the reservoir (Peters et al. 2005); more time and heat causes more of the original material to break down. Nevertheless, even the most thermally mature oil reservoir is not generally interpreted to be of abiotic origin (though some debates do persist).

Abiotic synthesis can often proceed by sequential addition of carbon atoms or $\mathrm{CH}_{2}$, thus yielding a relatively smooth Gaussian mass spectrum, but other synthesis pathways exist that can yield considerably more complex spectra. Here we examine two experiments that reveal some of the complexity that can emerge from abiotic chemistry, and some of difficulty in distinguishing that chemistry from biological processes.

In their analysis of lipid formation via Fischer-Tropsch-Type (FTT) reactions under hydrothermal conditions, McCollom et al. (1999) demonstrated facile production of long chain alkanoic acids and alcohols - compounds found in many biological membranes. Starting with oxalic or formic acid, FTT production of lipids proceeded to yield compounds containing over 35 carbon atoms $\left(>\mathrm{C}_{35}\right)$. To explain the abundance of acids and alcohols in their results, McCollom et al. (1999) argue that FTT synthesis proceeds through addition of a COO group to the end of a hydrocarbon followed by reduction to an alcohol. Alkenes can then be formed via dehydration of the alcohol and formation of the double bond. In other words, abiotic synthesis does generate organic subunits and in some cases those subunits can be polymerized.

Importantly, though distinct patterns persist in the mass spectra from FTT synthesis, McCollom et al. (1999) reported that differentiation from biogenic lipids was possible because, unlike biology, FTT synthesis yielded: 1) few isoprenoids, a common baseline biomarker for life on Earth (though not necessarily for life on other worlds), 2) few branched chain lipids, 3) no even/odd carbon number abundance in the alkane and alkene series, 4) an abundance of saturated $n$-alkanols and $n$-alkanoic acids, and 5) a decreasing abundance of heavy carbon compounds beyond $\mathrm{C}_{15}$.

FTT synthesis has long been appreciated as an important abiotic process for gas and liquid phase carbon reactions, but on worlds like Europa, the intense radiation environment and cold $(100 \mathrm{~K})$ icy surface provides an unfamiliar setting for organic chemistry. Hand and Carlson have recreated the surface environment of Europa in the lab, complete with a $100 \mathrm{keV}$ electron gun for irradiating ice mixtures (Hand 2007; Hand et al. 2007). Attached to the vacuum chamber is a quadrupole mass spectrometer used to scan organics up to an $\mathrm{m} / \mathrm{z}$ of 100 . Figure 1 shows the mass spectrum integrated over the warming 


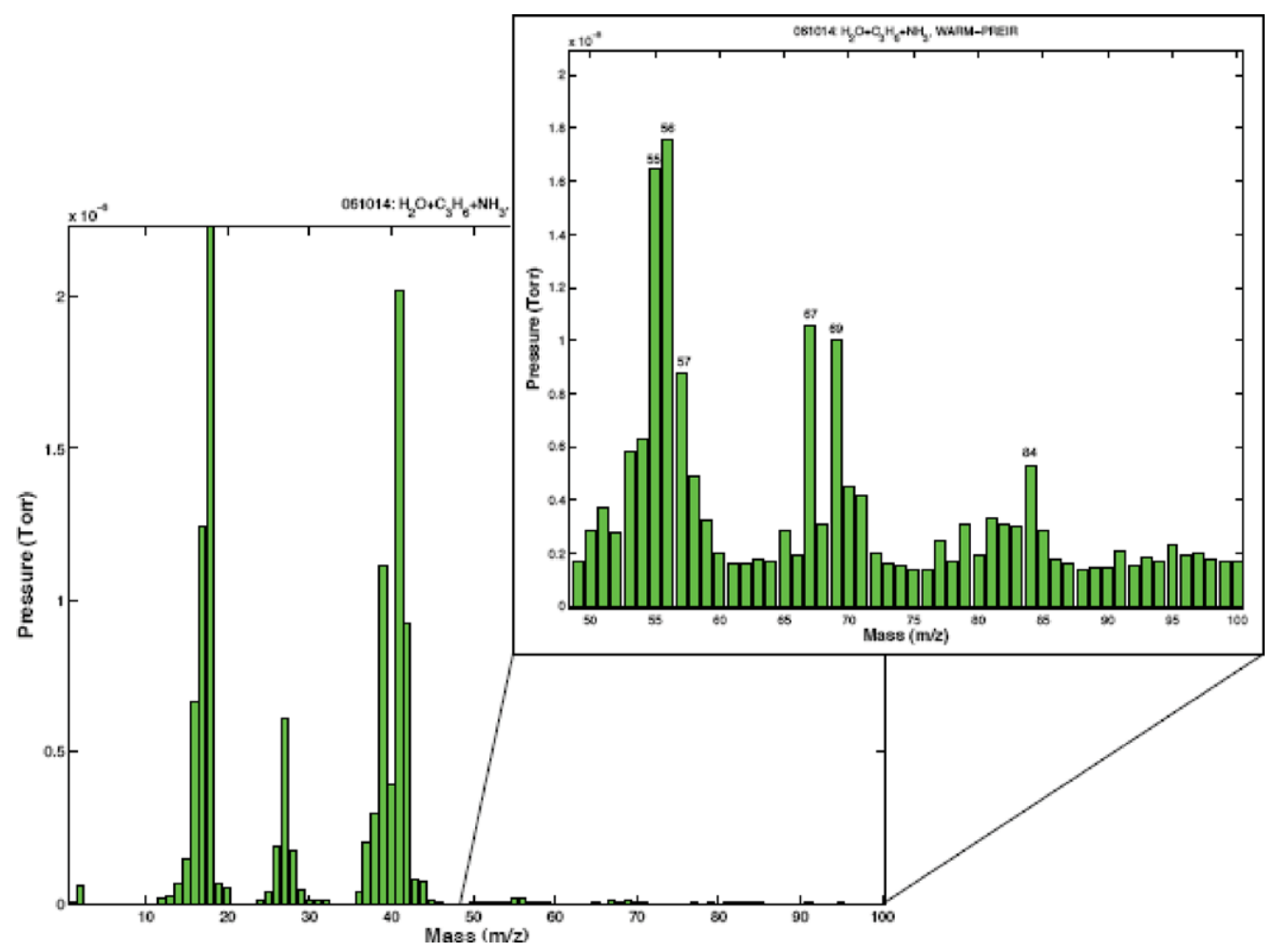

Figure 1. Mass spectrum results integrated over the warming period from 70-300 K after $10 \mathrm{keV}$ electron irradiation of a $\mathrm{H}_{2} \mathrm{O}+\mathrm{NH}_{3}+\mathrm{C}_{3} \mathrm{H}_{6}$ ice film (adapted from Hand and Carlson, in prep. The inset shows detail of the $\mathrm{m} / \mathrm{z}$ region 50-100. Abiotic radiolysis shows preferential formation of certain hydrocarbons, not just a broad distribution of all variations. This complicates the use of the 'Lego Principle' (McKay 2004) when working to differentiate abiotic chemistry from biological chemistry on icy worlds.

from $70 \mathrm{~K}$ to $300 \mathrm{~K}$ of products evolved from an irradiated $\mathrm{H}_{2} \mathrm{O}+\mathrm{NH}_{3}+\mathrm{C}_{3} \mathrm{H}_{6}$ ice film (modified from Hand and Carlson, in prep). Clearly mass spectra that extend to much larger $\mathrm{m} / \mathrm{z}$ are critical for astrobiology, but the spectrometer used in this work provides a useful comparison to flown spectrometers (e.g. on Cassini and Huygens).

The cluster of peaks around $\mathrm{m} / \mathrm{z}$ of 18 is from water and associated ions. At $\mathrm{m} / \mathrm{z}=27$ lies a strong peak from $\mathrm{HCN}$. Peaks at $\mathrm{m} / \mathrm{z}=41$ and 39 were likely due to the $\mathrm{OCN}^{-}$ion and to synthesized nitriles such as isocyanomethane $(\mathrm{C}-\mathrm{N} \equiv \mathrm{C})$ and acetonitrile $(\mathrm{C}-\mathrm{C} \equiv \mathrm{N})$. The peak at 39 is a related ion, HCNC.

At larger $\mathrm{m} / \mathrm{z}$, a cluster of peaks around $\mathrm{m} / \mathrm{z}=56$ was seen. Much of this was likely due to the nitrile of propane (isocyanopropane, $\mathrm{C}-\mathrm{C}-\mathrm{C} \equiv \mathrm{N}$ ). Hydrocarbons without nitrogen, such as propenal may have also contributed to this peak. For the heaviest ions, those at $\mathrm{m} / \mathrm{z}=67,69$, and 84 (Figure 1 inset), several complex nitriles serve as candidates. For the peak at 84 , formamide with a cyanomethyl- group is consistent with both the mass spectra and infrared data. Similarly, 2-cyanoacetamide contains similar bonds (though it hosts an $\mathrm{NH}_{2}$ group, unlike cyanomethylformamide) and yields an $\mathrm{m} / \mathrm{z}$ of 84 . Loss of an $\mathrm{NH}, \mathrm{NH}_{2}$, or $\mathrm{NH}_{3}$ would then yield the peaks at 67 and 69 .

The critical observation for the present manuscript is that complexity exists even within the low mass range of this radiolysis experiment. The pattern of subunit synthesis is difficult to distinguish in this range, but based on matrix assisted laser desorption ionization analyses of the refractory residue left on the irradiated target, Hand and 
Carlson (in prep) argue for polymers of isopropyl-propionate $\left(\mathrm{C}_{6} \mathrm{H}_{12} \mathrm{O}_{2}\right)$. In a similar experiment, Bernstein et al. (1995) reported synthesis of hexamethylenetetramine (HMT, or $\mathrm{C}_{6} \mathrm{H}_{12} \mathrm{~N}_{4}$ ) in their GCMS analysis of residues resulting from UV-photolysis of $\mathrm{H}_{2} \mathrm{O}+$ $\mathrm{CH}_{3} \mathrm{OH}+\mathrm{CO}+\mathrm{NH}_{3}$ ice. Again, the observation was that abiotic synthesis can lead to preferential formation of select compounds.

As per McCollom et al. (1999), information within the structure of the mass spectra can often be used to distinguish abiotic from biogenic, at least for the case of known biochemistry. However, for carbon-based life on distant worlds, the lack of a prior knowledge of a preferential organic biochemistry could lead to false positives in environments where abiotic synthesis leads to complex patterns of organic polymers.

\section{Spectroscopic Signatures}

The elucidation of spectroscopic biosignatures has been the subject of considerable work, however, much of that work has focused on the gas phase, i.e. planetary atmospheres (des Marais et al. 2002; Pilcher 2003; Kiang et al. 2007b,a). Atmospheric biosignatures largely fall into two categories, 1) the detection of species in chemical disequilibrium, and 2) isotopic fractionation. Though of great utility for the study of worlds like Mars, Venus, and extrasolar planets, gas-phase biosignatures are generally not applicable to the vacuum environments present on the surfaces of many icy worlds. Indeed, at Europa the thin, radiolytically produced oxygen atmosphere (Hall et al. 1995) serves as an important reminder of the potential for false positives when employing the chemical disequilibrium argument.

For spectroscopic biosignatures of material in the solid phase, e.g. ices, work has been largely limited to pigments such as chlorophyl-a, chlorophyl-b, carotenoids, and other chromophore molecules (Painter et al. 2001). Blooms of the the algae C. nivalis, also known as 'snow algae', show a reflectance spectrum with absorption by carotenoids in the range $0.4-0.64 \mu \mathrm{m}$ and a local reflectance minimum at $0.68 \mu \mathrm{m}$ due to absorption by chlorophyll-a and -b. Using ground calibration, the depth and breadth of the $0.68-\mu \mathrm{m}$ feature was found to increase with increasing algal concentration, permitting mapping of algal concentrations in the snowpack from $20 \mathrm{~km}$ altitude (Painter et al. 2001).

The detection threshold for algal concentrations was determined to be $\sim 2,300$ cells $\mathrm{ml}^{-1}$. Assuming that measurments of algal concentrations represented the mean for the top $10 \mathrm{~cm}$, Painter et al. (2001) estimated the total biomass to be $16.55 \mathrm{~kg}$ for the $\sim 0.75$ $\mathrm{km}^{2}$ region surveyed.

The detection threshold of Painter et al. (2001) is two orders of magnitude below the concentration of algae in the sea surface and over three orders of magnitude below that of microbes in hot springs and hydrothermal vents (Hand et al. 2009). Were life on Europa to contain biological pigments comparable to chlorophyll and carotenoids, detection from orbit at concentrations far below terrestrial oceanic concentration is feasible. Without such compounds, however, prospects for detection from orbit are very limited.

Spectral bands associated with the covalent bonds of biological structures are not as strong as the absorptions due to pigments and chromophores. Nevertheless, the systematic detection and mapping of these weaker spectral features will be our first tool for assessing biosignatures on the surfaces of icy worlds. In the sections that follow we divide spectroscopic features into 'Spectroscopic Signposts', 'Compelling Chemistry', and finally 'Complexity'. By 'spectroscopic signposts' we mean strong spectral features that are due to compounds that could be associated with biological processes. The strength of the feature makes it easy to see and the compound or bond revealed by the feature provides some indication that further examination of the region may be warranted. The features deemed as indicative of 'compelling chemistry' are those resulting from bonds 
between biologically essential elements such as carbon, nitrogen, phosphorous and sulfur. Finally, the features that fall into the 'complexity' class are those spectral features that result from added structural complexity in a molecule, e.g. shifts in the band position of a functional group resulting from polymerization and refinements of the vibrational modes available to the functional group in polymeric form.

\subsection{Spectroscopic Signposts}

Carbon dioxide has a very strong band at $4.27 \mu \mathrm{m}$ and though $\mathrm{CO}_{2}$ is not in and of itself a biosignature, this band can be used as an important sign post for carbon chemistry. When organic matter is exposed to the radiation environment of Europa's surface, some of that material is destroyed and some of the liberated carbon goes into $\mathrm{CO}_{2}$. By mapping the geographic distribution of this spectral feature we will be able to generate a broad map of surface regions containing carbon chemistry, and possibly biochemistry.

The first spectroscopic clue toward detection of organic chemistry on Europa will be the $\sim 3.44 \mu \mathrm{m}$ bands of the $\mathrm{C}-\mathrm{H}$ stretch. This feature appears on the shoulder of the broad $3 \mu \mathrm{m}$ water band and has been detected on Ganymede, Callisto, Enceladus, and comets (McCord et al. 1997; Brown et al. 2006; Chyba and Sagan 1987; A'Hearn et al. 2005). Both abiotic and biological hydrocarbons show this feature, but detection of this feature provides the basis for searching for weaker signatures indicative of carbon chemistry.

The second and third overtones of the $\mathrm{C}-\mathrm{H}$ stretch $\left(2 \nu_{3}\right.$ and $\left.3 \nu_{3}\right)$ for organics in ice occur at $1.67 \mu \mathrm{m}$ and $1.19 \mu \mathrm{m}$ respectively (Clark et al., in press). Features of methane and ethane in ice occur between $2.27-2.46 \mu \mathrm{m}$. Methane has a stronger line at $7.67 \mu \mathrm{m}$ and since methane is a by product of many chemosynthetic ecosystems on Earth, detection of methane features serves as a useful signpost. Finally, lipids and proteins display C-H absorption features near $2.3 \mu \mathrm{m}$ (Dalton et al. 2003).

After water and carbon, nitrogen is often considered to be the next key elemental signpost for habitability and life detection (Mancinelli and Banin 2004; Capone et al. 2006). The weak N-H band at $\sim 3.1 \mu \mathrm{m}$ is important to look for, but it overlaps with the broad water feature at $3 \mu \mathrm{m}$. The triple bond of carbon to nitrogen yields a strong band in the 4.56-4.60 $\mu \mathrm{m}$ region and this may be the best band for identifying the presence of nitrogen chemistry. The band occurs in abiotic radiolyitc chemistry experiments, as well as on planetary and astrophysical bodies, including Ganymede and Callisto (McCord et al. 1997).

\subsection{Compelling Chemistry}

After mapping strong and relatively easy features to identify, the next step is to search for features that can be associated with biochemistry. The carbonyl bond $(\mathrm{C}=\mathrm{O})$ has a strong band that appears at $\sim 5.9 \mu \mathrm{m}$ and is associated with carbonic acid, esters, and aldehydes, all of which can be produced abiotically under europan conditions (see e.g. Hand et al. (2007)). If observed at slightly longer wavelengths $(5.83-5.95 \mu \mathrm{m})$ then this band can be associated with the carbonyl stretch in nucleic acids (Naumann et al. 1996; Maquelin et al. 2002). The shift in position results from the ordered structure of biopolymers and is discussed in more detail below.

Detection of carbon bonded to carbon is clearly of astrobiological interest. The C-C $\sigma$-bond shows several features in the region $6.62-7.37 \mu \mathrm{m}$. The strongest features are at $6.82 \mu \mathrm{m}$ and $7.25 \mu \mathrm{m}$ and have very little overlap with water features.

The thiol functional group (-S-H attached to carbon) is important for metabolic and structural components (De Duve 2005). Mapping of thiols and any association with carbon and nitrogen features could serve to differentiate endogenous biologically utilized sulfur from either exogenous radiolytically processed sulfur or endogenous sulfate salts. 


\subsection{Complexity}

Complexity in spectroscopic analysis here refers to complex bonds and shifts in band position due to polymerization and extended molecular backbones. The $\pi$-bond $(\mathrm{C}=\mathrm{C})$ of a carbon-carbon linkage results in a strong band at $6.08 \mu \mathrm{m}$. Alkenes in ice show this feature, as well as several weaker lines in the region of $6.9 \mu \mathrm{m}$. The $\pi$-bond is easily destroyed by irradiation, causing many of these bands to disappear or be greatly reduced in strength (Hand 2007). Detection of the $6.08 \mu \mathrm{m}$ feature could be interpreted as complex endogenous organic chemistry on a geologically young surface.

The $\mathrm{C}-\mathrm{N}$ bond, and bands associated with the amide structure $(\mathrm{O}=\mathrm{C}-\mathrm{N}-\mathrm{H})$, provide strong potential for spectroscopic biosignatures. Dalton et al. (2003) froze samples of the microbes Sulfolobus shibatae, Deinococcus radiodurans, and Escherichia coli to characterize spectral signatures under Europan conditions of vacuum and low temperature $(100 \mathrm{~K})$, funding that the 2.05 and $2.17 \mu \mathrm{m} \mathrm{C}-\mathrm{N}$ bands of proteins were subtle but distinguishable in the laboratory.

Experiments by Hand and Carlson (Hand 2007; Hand et al. 2009) in Europa simulation chambers have shown that electron irradiated spores of Bacillis pumilus can be spectroscopically distinguished from the products of abiotic radiolysis. It is important to note that the total dose in these experiments was sufficient to disrupt every bond in the sample. The distinguishing features arise from shifts in the amide bands associated with amino acids. Amino acids in proteins are arranged in ordered sheets and helical structures resulting in a shift of the band position. Amino acids and other amide structures produced radiolytically do not have this added layer of structural complexity.

The amide I band of $\alpha$-helical structures in proteins occurs at $6.04 \mu \mathrm{m}$ and results from the carbonyl bond in the right-handed spiral of the helical structure. In abiotic radiolysis experiments this band was seen at $5.99 \mu \mathrm{m}$. The amide I band seen in $\beta$-pleated sheets of proteins occurs at $6.11 \mu \mathrm{m}$ (Naumann et al. 1996), and is also due to the carbonyl stretch interacting with the amide $\mathrm{N}-\mathrm{H}$, but in $\beta$-pleated sheets the strands of linked amino acids form a sheet by hydrogen bonding with neighboring strands. This difference in structural geometry shifts the amide $\mathrm{I}$ in $\beta$-sheets to the longer wavelength.

The amide II bond was also seen by Hand and Carlson to persit with subtle but distinguishable differences between the abiotic and biological radiolysis experiments. The amide II band comes from bending of $\mathrm{N}-\mathrm{H}$ coupled to $\mathrm{C}-\mathrm{N}$ stretch (Hayashi and Mukamel 2007). In the irradiated biological samples the band was seen at $6.5 \mu \mathrm{m}$, whereas in the abiotic samples it was seen at at $6.27 \mu \mathrm{m}$. In the abiotic material the feature results from $\mathrm{NH}_{2}$ scissoring and uncoordinated amide bonds.

Detection and mapping of the above described amide and phosphodiester features could yield clues toward the detection of complex organic chemistry associated with structures of known importance to life here on Earth. Working through the complete set of spectral signposts, features of compelling chemistry, and those indicative of complexity could point the way toward possible biochemistry on icy worlds. Mapping the spatial distribution of these spectroscopic features across geological fractures, ridges, and chaos terrain could permit differentiation between endogenous organic chemistry in the subsurface and organic chemistry driven by surface radiolysis and exogenous delivery.

\section{Conclusions}

The ability to differentiate abiotic organic material from material of a biological origin is a critical task for astrobiology. Mass spectrometry and spectroscopy provide key tools for advancing this task and are two techniques that provide useful and highly 
complementary compositional information independent of a specific biochemical pathway (e.g. DNA-RNA-Proteins).

Patterns in the mass spectrometric distribution of organics can, to first order, be applied to differentiate abiotic from biogenic, as per the 'Lego Principle' (McKay 2004). However, much caution is warranted as both Fischer-Tropsch Type synthesis and radiolytically driven chemistry can lead to complex mass spectra. For terrestrial biochemistry, our knowledge of the lipids and proteins utilized by biology make it possible to use additional metrics within a mass spectrum to distinguish abiotic from biogenic material (McCollom et al. 1999). The biochemistry of an alien ecosystem may reveal important clues in the pattern and complexity of a mass spectrum, but the lack of an a priori comparison will limit the utility of the 'Lego Principle.'

The analyses made possible by infrared spectroscopy reveal composition (e.g. atoms of $\mathrm{C}, \mathrm{H}, \mathrm{N}, \mathrm{O}, \mathrm{P}, \mathrm{S}$ ), connectivity (e.g. single, double, or triple bonds) and concentration. Though compositional information and geographic mapping of concentrations will provide an important context for astrobiology, the complexity revealed by functional group connectivity is arguably the most useful information. The bonds associated with the polymers of life, such as amides of proteins and phosphodiesters of nucleic acids, will serve as powerful spectroscopic biosignatures, especially if coupled to mass spectra consistent with such polymerization.

Finally, we note that the detection of life from orbit - by a spacecraft designed for studying the Jovian system - is not unprecedented. During the Galileo spacecraft's gravity assist fly-by of the Earth, Sagan et al. (1993) turned the instrument payload toward Earth and detected several signs of life, including an atmosphere out of chemical equilibrium and regions of the surface rich with photosynthetic pigments.

Though it was the Earth being observed, the Galileo experiment provided a useful proof-of-concept: we are technologically capable of detecting life on an inhabited planet. However, as Sagan et al. (1993) wisely advised, as we move forward with our search for life beyond Earth, we must use caution and invoke life only as 'the hypothesis of last resort'.

\section{Acknowledgement}

The research by K. P. Hand was carried out at the Jet Propulsion Laboratory, California Institute of Technology, under a contract with the National Aeronautics and Space Administration. Government sponsorship acknowledged.

\section{References}

A'Hearn, M. S., Belton, M. S. J., Delamere, W. A., Kissel, J., Klaasen, K. P., McFadden, L. A., Meech, K. J., Melosh, H. J., Schultz, P. H., Sunshine, J. M. et al. 2005, Deep Impact: Excavating Comet Tempel 1. Science, 310(5746):258-264

Anderson, J. D., Schubert, G., Jacobson, R. A., Lau, E. L., Moore, W. B., \& Sjorgen, W. L. 1998, Europa's Differentiated Internal Structure: Inferences from Four Galileo Encounters. Science, 281(5385):2019-2022

Benner, S. A., Devine, K. G., Matveeva, L. N., \& Powell, D. H. 2000, The missing organic molecules on mars. Proceedings of the National Academy of Sciences, 97(6):2425

Bernstein, M. P., Sandford, S. A., Allamandola, L. J., Chang, S., \& Scharberg, M. A. 1995, Organic Compounds Produced by Photolysis of Realistic Interstellar and Cometary Ice Analogs Containing Methanol. The Astrophysical Journal, 454:327

Biemann, K., Oro, J., Toulmin III, P., Orgel, L. E., Nier, A. O., Anderson, D. M., Flory, D., Diaz, A. V., Rushneck, D. R., \& Simmonds, P. G. 1977, The search for organic substances and inorganic volatile compounds in the surface of mars. Journal of Geophysical Research, 82 
Brown, R. H., Clark, R. N., Buratti, B. J., Cruikshank, D. P., Barnes, J. W., Mastrapa, R. M. E., Bauer, J., Newman, S., Momary, T., Baines, K. H., et al. 2006, Composition and Physical Properties of Enceladus' Surface. Science, 311(5766):1425-1428

Capone, D. G., Popa, R., Flood, B., \& Nealson, K. H. 2006, Follow the nitrogen. Science (Washington, D. C.), 312(5774):708-709

Carlson, R. W., Calvin, W. M., Dalton III, J. B., Hansen, G. B., Hudson, R. L., Johnson, R. E., McCord, T. B., \& Moore, M. H. 2009, Europa, chapter Europa's Surface Composition, pages 283-327. University of Arizona Press

Chyba, C. \& Sagan, C. 1987, Infrared emission by organic grains in the coma of comet Halley. Nature, 330(6146):350-353

Chyba, C. F. 2000, Energy for microbial life on Europa. Nature, 403(6768):381-2

Chyba, C. F. \& McDonald, G. D. 1995, The Origin of Life in the Solar System: Current Issues. Annual Review of Earth and Planetary Sciences, 23(1):215-249

Cleland, C. E. \& Chyba, C. F. 2002, Defining 'life'. Origins of Life and Evolution of Biospheres, 32(4):387-393

Cooper, J. F., Johnson, R. E., Mauk, B. H., Garrett, H. B., \& Gehrels, N. 2001, Energetic Ion and Electron Irradiation of the Icy Galilean Satellites. Icarus, 149:133-159

Dalton, J. B., Mogul, R., Kagawa, H. K., Chan, S. L., \& Jamieson, C. S. 2003, Near-infrared detection of potential evidence for microscopic organisms on Europa. Astrobiology, 3(3): 505-29

De Duve, C. 2005, Singularities: Landmarks on the Pathways of Life. Cambridge University Press

des Marais, D. J., Harwit, M. O., Jucks, K. W., Kasting, J. F., Lin, D. N. C., Lunine, J. I., Schneider, J., Seager, S., Traub, W. A., \& Woolf, N. J. 2002, Remote sensing of planetary properties and biosignatures on extrasolar terrestrial planets. Astrobiology, 2(2):153-181

Figueredo, P. H., Greeley, R., Neuer, S., Irwin, L., \& Schulze-Makuch, D. 2003, Locating Potential Biosignatures on Europa from Surface Geology Observations. Astrobiology, 3(4): $851-861$

Greeley, R., Chyba, C. F., Head III, J. W., McCord, T. B., McKinnon, W. B., Pappalardo, R. T., \& Figueredo, P. H. 2004, Geology of Europa. In F. Bagenal, T. E. Dowling, and W. B. McKinnon, editors, Jupiter. The planet, satellites and magnetosphere, chapter 15, pages 329-362. Cambridge University Press, first edition

Hall, D. T., Strobel, D. F., Feldman, P. D., McGrath, M. A., \& Weaver, H. A. 1995 Detection of an oxygen atmosphere on Jupiter's moon Europa. Nature, 373(6516):677-679

Hand, K. P. 2007, On the Physics and Chemistry of the Ice Shell and Subsurface Ocean of Europa. PhD thesis, PhD Thesis, Department of Geological and Environmental Sciences, Stanford University, Stanford, CA

Hand, K. P. \& Chyba, C. F. 2007, Empirical constraints on the salinity of the europan ocean and implications for a thin ice shell. Icarus, 189(2):424-438

Hand, K. P., Carlson, R. W., \& Chyba, C. F. 2007, Energy, chemical disequillibrium, and geological constraints Europa. Astrobiology, 7(6):1006-1022

Hand, K. P., Chyba, C. F., Priscu, J. C., Carlson, R. W., \& Nealson, K. H. 2009 Europa, chapter Astrobiology and the Potential for Life on Europa, pages 589-629. University of Arizona Press

Hayashi, T. \& Mukamel, S. 2007, Vibrational-Exciton Couplings for the Amide I, II, III, and A Modes of Peptides. J. Phys. Chem. B, 10.1021/jp070369b(111):11032-11046

Joyce, G. F. 1995, The RNA world: Life before DNA and protein. In B. Zuckerman and M. Hart, editors, Extraterrestrials: Where are they?, pages 139-151. Cambridge University Press

Kargel, J. S., Kaye, J. Z., Head, J. W., Marion, G. M., Sassen, R., Crowley, J. K., Ballesteros, O. P., Grant, S. A., \& Hogenboom, D. L. 2000, Europa's Crust and Ocean: Origin, Composition, and the Prospects for Life. Icarus, 148(1):226-265

Khurana, K. K., Kivelson, M. G., Stevenson, D. J., Schubert, G., Russell, C. T., Walker, R. J., \& Polanskey, C. 1998, Induced magnetic fields as evidence for subsurface oceans in Europa and Callisto. Nature, 395(6704):749-751

Kiang, N. Y., Segura, A., Tinetti, G., Blankenship, R. E., Cohen, M., Siefert, J., Crisp, D., \& Meadows, V. S. 2007, pectral signatures of photosynthesis. ii. coevolution with other stars and the atmosphere on extrasolar worlds. Astrobiology, 7(1):252-274 
Kiang, N. Y., Siefert, J., \& Govindjee, B. R. E. 2007, Spectral signatures of photosynthesis. i. Astrobiology, 7(1):222-251

Mancinelli, R. L. \& Banin, A. 2004, Where is the nitrogen on Mars? International Journal of Astrobiology, 2(03):217-225

Maquelin, K., Kirschner, C., Choo-Smith, L. P., van den Braak, N., Endtz, H., Naumann, D., \& Puppels, J. P. 2002, Identification of medically relevant microorganisms by vibrational spectroscopy. Journal of Microbiological Methods, 51(3):255-271

McCollom, T. M. 1999, Methanogenesis as a potential source of chemical energy for primary biomass production by autotrophic organisms in hydrothermal systems on Europa. Journal of Geophysical Research, 104(E12):30729-30742

McCollom, T. M., Ritter, G., \& Simoneit, B. R. T. 1999, Lipid synthesis under hydrothermal conditions by fischer-tropsch-type reactions. Origins of Life and Evolution of Biospheres, 29(2):153-166

McCord, T. B., Carlson, R. W., Smythe, W. D., Hansen, G. B., Clark, R. N., Hibbitts, C. A., Fanale, F. P., Granahan, J. C., Segura, M., Matson, D. L., et al. 1997, Organics and other molecules in the surfaces of Callisto and Ganymede. Science, 278(5336):271-275

McCord, T. B., Hansen, G. B., Martin, P. D., \& Hibbitts, C. 1998, Non-water-ice constituents in the surface material of the icy Galilean satellites from the Galileo near-infrared mapping spectrometer investigation. Journal of Geophysical Research, 103(E4):8603-8626

McKay, C. P. 2004, What is life-and how do we search for it in other worlds? PLoS Biology, $2(9)$

Naumann, D., Schultz, C. P., \& Helm, D. 1996, What can infrared spectroscopy tell us about the structure and composition of intact bacterial cells? In H. H. Mantsch and D. Chapman, editors, Infrared Spectroscopy of Biomolecules, pages 279-310. Wiley-Liss: New York

NRC. New Frontiers in the Solar System an Integrated Exploration Strategy. National Academies Press, 2003.

Painter, T. H., Duval, B., Thomas, W. H., Mendez, M., Heintzelman, S., \& Dozier, J. 2001, Detection and Quantification of Snow Algae with an Airborne Imaging Spectrometer. Applied and Environmental Microbiology, 67(11):5267-5272

Pappalardo, R. T. \& Head III, J. W. 2001, The Thick-Shell Model of Europa's Geology: Implications for Crustal Processes. 32nd Annual Lunar and Planetary Science Conference, March 12-16, 2001, Houston, Texas, abstract no. 1866

Pappalardo, R. T., Head, J. W., Greeley, R., Sullivan, R. J., Pilcher, C., Schubert, G., Moore, W. B., Carr, M. H., Moore, J. M., Belton, M. J. et al. 1998 Geological evidence for solid-state convection in Europa's ice shell. Nature, 391(6665):365-368

Peters, K. E., Walters, C. C., \& Moldowan, J. M. 2005, The Biomarker Guide Vol. II: Biomarkers and isotopes in petroleum systems and Earth history. Cambridge University Press

Phillips, C. B., McEwen, A. S., Hoppa, G. V., Fagents, S. A., Greeley, R., Klemaszewski, J. E., Pappalardo, R. T., Klaasen, K. P., \& Breneman, H. H. 2000, The search for current geologic activity on Europa. Journal of Geophysical Research, 105(E9):22579-22598

Pilcher, C. B. 2003, Biosignatures of early earths. Astrobiology, 3(3):471-486

Sagan, C., Thompson, W. R., Carlson, R., Gurnett, D., \& Hord, C. 1993, A search for life on Earth from the Galileo spacecraft. Nature, 365(6448):715-721

Schubert, G., Anderson, J. D., Spohn, T., \& McKinnon, W. B. 2004, Interior composition, structure and dynamics of the Galilean satellites, pages 281-306. Cambirdge University Press

Summons, R. E., Albrecht, P., McDonald, G., \& Moldowan, J. M. 2008, Molecular biosignatures. Space Science Reviews, 135(1):133-159

Zimmer, C., Khurana, K. K., \& Kivelson, M. G. 2000, Subsurface oceans on Europa and Callisto: constraints from Galileo magnetometer observations. Icarus, 147(2):329-347

Zolotov, M. Y. \& Shock, E. L. 2004 A model for low-temperature biogeochemistry of sulfur, carbon, and iron on Europa. J. Geophys. Res, 109:2003JE002194 